\title{
Correction to: Complications in Posterosuperior and Three Tendon Rotator Cuff Repair
}

Stephan Pauly and Markus Scheibel

\section{Correction to:}

L. Lafosse et. al. (ed.), Complications in Arthroscopic Shoulder Surgery, https://doi.org/10.1007/978-3-030-24574-0_8

This chapter was inadvertently published with incorrect Author name as Stefan Pauly and now it is corrected as Stephan Pauly at every occurrence in the Book. 